\title{
Aprendizaje cooperativo y la formación docente por competencias en la Universidad Nacional Daniel Alcides Carrión
}

\section{Yanapaanakuy yaćhana yaćhachita yaćhachi atipanakunawan Daniel Alcides Carrión hatun yaćhaywasi suyuykunaćhu}

\section{Ogotagantsi omitokotabagima aike kara inganëma ogomeantatsi okokagaigiri ora ogomentotsipongo universidad Nacional Daniel Alcides Carrión}

Recibido: 22 diciembre 2019 Aprobado: 22 mayo 2020

\author{
Teófilo Félix Valentín Melgarejo \\ Nacionalidad: Peruana \\ Filiación: Universidad Nacional Daniel Alcides Carrión \\ Correo: tvalentinm@undac.edu.pe \\ ORCID: https://orcid.org/oooo-0002-4063-5516
}

\section{Resumen}

El presente trabajo, tuvo la intención de establecer la relación entre aprendizaje cooperativo y la formación docente por competencias en la Universidad Nacional Daniel Alcides Carrión, en la investigación se aplicó el método científico de tipo básico y nivel descriptivo, con diseño correlacional; además, se trabajó con una muestra de tipo no probabilística de 86 estudiantes de la Facultad de Ciencias de la Educación, en dicho estudio el cálculo de correlación rs $=0.648>0$; esto significa que, existe relación significativa entre aprendizaje cooperativo y la formación docente por competencias en la Universidad Nacional Daniel Alcides Carrión.

\section{Palabras clave:}

Aprendizaje cooperativo, formación docente, competencias, metodología.

\section{Lisichiku limaykuna:}

Yanapaanakuy yaćhana, yaćhachikuna yaćhachi, atipanakuna, yaćhanap

Nibarintsipage katingatsaro: Ogotagantsi omitokotobakanero, ikanta ogomeantasinera, iraretagekimara, ingankerori.

\section{Datos del autor}

Teófilo Félix Valentín Melgarejo es docente e investigador en educación, Título Profesional en Lengua y Literatura. Maestría en Educación - Mención: Didáctica y Tecnología de la Información. Doctor en Ciencias de la Educación, obtenidos en la Universidad Nacional Daniel Alcides Carrión. Post Doctorado en Ciencias en la Universidad Nacional Hermilio Valdizán. 


\section{Cooperative Learning and Teacher Training by Competencies at the National University Daniel Alcides Carrión}

\begin{abstract}
The present work, had the intention of setting the relation between cooperative learning and teacher training by competencies at National University Daniel Alcides Carrión, in the research he was applied the scientific basic kind method and descriptive level with design, correlates; besides, he worked one with a not probabilistic sample of kind of 86 students of Empower her of Education Sciences, in saying I study the $=0$ rs correlation calculation. $648>0$; this means that, significant relation exists enter cooperative learning and the educational education by competences in National University Daniel Alcides Carrión.
\end{abstract}

\section{Keywords}

Cooperative learning, teacher training, competencies, methodology.

\section{Aprendizagem cooperativa e a formação docente por competências na Universidade Nacional Daniel Alcides Carrión}

\section{Resumo}

O presente trabalho, teve a intenção de estabelecer a relação entre a aprendizagem cooperativa e a formação docente por competências na Universidade Nacional Daniel Alcides Carrión, na pesquisa foi aplicado o método básico científico e o nível descritivo com o desenho de correlacional; além disso, trabalhou-se com uma amostra não probabilística de 86 estudantes do Curso de Ciências da Educação em dito estudo o cálculo de correlação rs = o rs. 648 >o; isso significa que, existe uma relação significativa entre na aprendizagem cooperativa e a formação docente por competências na Universidade Nacional Daniel Alcides Carrión.

\section{Palavras-chave:}

Aprendizagem cooperativa, formação docente, competências, metodologia. 


\section{Introducción}

Actualmente se viene modificando las formas de producción del conocimiento, de un conocimiento pasivo, espontáneo, poco significativo y transmitido; a un conocimiento activo, duradero, significativo y por descubrimiento para la obtención de un nuevo saber. Por ello, resulta fundamental el conocimiento y aplicación de metodologías que favorezcan la participación activa de los estudiantes y promueva el desarrollo de la capacidad para razonar de forma crítica.

Además, la compleja realidad educativa actual requiere la aplicación de métodos innovadores de cooperación que permitan despertar la curiosidad y creatividad del estudiante universitario para así hacer más fácil el aprendizaje; al respecto, el aprendizaje cooperativo es una metodología que favorece la participación activa de los estudiantes y promueve el desarrollo de la capacidad de razonar de forma crítica; asimismo, los cambios generados en el sistema educativo, como consecuencia de las políticas educativas, se vieron en la necesidad de reformar modelos educativos basados en competencias, desde educación básica hasta educación superior. Para responder a estas exigencias; nuevas metodologías, estrategias y técnicas de aprendizaje fueron incorporadas al sistema educativo, siendo uno de ellos el aprendizaje cooperativo, que permite desarrollar y mejorar competencias de manera integral.

La Facultad de Ciencias de la Educación de la Universidad Nacional Daniel Alcides Carrión, hace que los procesos formativos se centren en el aprendizaje de los estudiantes y más concretamente en los resultados de aprendizaje expresados en términos de competencias, de manera integral para desarrollar habilidades cognitivas, intrapersonal e interpersonal; por ello, resulta fundamental el conocimiento y aplicación de metodologías que favorezcan la participación activa de los estudiantes y promuevan el desarrollo de la capacidad para razonar de forma crítica.

Teniendo en cuenta que en estos tiempos la formación docente por competencia es muy importante para el desenvolvimiento profesional del futuro docente; por esta razón, se requiere que el docente utilice estrategias metodológicas adecuadas y pertinentes ante una actividad de aprendizaje, ante esta necesidad los futuros docentes no lo están dando la importancia pertinente a las estrategia metodológicas durante su formación profesional; además, hay un desconocimiento sobre la relevancia e importancia de la metodología del aprendizaje cooperativo y su relación con respecto al desarrollo de habilidades cognitivas, interpersonales e intrapersonales de la formación docente por competencia. Para responder a estas situaciones problemáticas se planteó como objetivo general de la investigación, determinar el nivel de relación que existe entre el aprendizaje cooperativo y la formación docente por competencias en la Universidad Nacional Daniel Alcides Carrión, para lograr este objetivo se tuvo que describir el nivel de relación que existe entre el aprendizaje cooperativo con las competencias y habilidades cognitivas, intrapersonales e interpersonales.

Desde luego, existen trabajos referidos al tema de estudio que permite dar el sustento con sus aportes a la situación problemática y objetivos considerados. Ceinos y García (2009), en sus conclusiones mencionan, que:

El aprendizaje cooperativo debe tener una transferencia a la acción, no limitándose a ser un contenido teórico más. Esta técnica implica relaciones de interdependencia positiva entre el alumnado, así como un replanteamiento del papel del profesorado, el cual ha de estar preparado para asumir las dificultades iniciales que plantea el aprendizaje cooperativo. (p. 979). 
En la investigación sobre cómo utilizar el aprendizaje cooperativo para evaluar las competencias emocionales de los estudiantes: un ejemplo práctico en el nivel terciario, en sus conclusiones hace referencia, que cuando los estudiantes trabajan de manera cooperativa en una asignatura de idioma extranjero, no solo aprenden contenidos porque las actividades cooperativas requieren que los estudiantes participen activamente en el proceso de enseñanza-aprendizaje y que adquieran las competencias que necesitarán en el mercado laboral. En consecuencia, para cumplir con las actividades de cooperación preparadas para el tema Idioma inglés IV, como presentaciones orales y debates, los estudiantes deben negociar, comunicarse, ser críticos con las tareas, cooperar y resolver posibles problemas en los grupos, entre otras competencias emocionales. (Martínez, 2016, p. 161).

En la investigación sobre el aprendizaje cooperativo y las competencias genéricas en el estudiante de la Universidad Nacional Mayor de San Marcos, Lima 2017, en sus conclusiones hace referencia que existe relación positiva entre aprendizaje cooperativo y competencias genéricas; además, menciona que existe relación entre interdependencia positiva, la responsabilidad individual y grupal, la interacción estimuladora y las técnicas interpersonales del equipo con las competencias genéricas. (Carbajal, 2017, pp. 113-115).

Al respecto, el aprendizaje cooperativo es un enfoque que trata de organizar las actividades dentro del aula para convertirlas en una experiencia social y académica, en el cual los estudiantes trabajan en grupo para realizar las tareas de manera colectiva. El aprendizaje en este enfoque depende del intercambio de información entre los estudiantes, los cuales están motivados tanto para lograr su propio aprendizaje como para acrecentar los logros de los demás dentro del aula a partir de la interacción y la ayuda de forma sistemática.

Por otra parte, la formación docente por competencias es un proceso que enfrenta al alumno a tareas diversas de la vida profesional y social correspondientes al ejercicio de una profesión, en este caso la de ser docente, estimula un aprendizaje activo y contextualizado, fomenta la autonomía del alumno y eleva considerablemente su motivación al aproximar el aprendizaje que se promueve en las universidades con respecto a las competencias de carácter general y específicas para su posterior practica en su desempeño docente, respondiendo a las características de las instituciones educativas y su contexto. Puesto que la competencia en términos generales es multidimensional e incluye distintos niveles como saber (datos, conceptos, conocimientos), saber hacer (habilidades, destrezas, métodos de actuación), saber ser (actitudes y valores que guían el comportamiento) y saber estar o convivir (capacidades relacionada con la comunicación interpersonal y el trabajo cooperativo); en otras palabras, la competencia es la capacidad de un buen desempeño en contextos complejos y auténticos. Se basa en la integración y activación de conocimientos, habilidades, destrezas, actitudes y valores.

Para llegar a entender con mayor claridad el tema de estudio, es importante el análisis de las variables involucradas y la explicación de cada uno de ellas desde diferentes fuentes de información.

El Aprendizaje Cooperativo, según Rué (2012):

Es un conjunto de procedimientos de enseñanza que parten de la organización de la clase en pequeños grupos mixtos y heterogéneos donde los alumnos trabajan conjuntamente de forma coordinada entre sí para resolver tareas académicas y profundizar en su propio aprendizaje. (p. 1). 
Por lo que, un grupo cooperativo tiene un sentido de responsabilidad individual, lo cual significa que cada uno es partícipe de la tarea propia y de los demás, entendiendo que su trabajo es imprescindible para el éxito del grupo.

El aprendizaje cooperativo implica que los estudiantes trabajen juntos para realizar actividades de aprendizaje. Johnson, Johnson y Holubec (1999) afirma que:

La cooperación consiste en trabajar juntos para alcanzar objetivos comunes. En una situación cooperativa, los individuos procuran obtener resultados que sean beneficiosos para ellos mismos y para todos los demás miembros del grupo. El aprendizaje cooperativo es el empleo didáctico de grupos reducidos en los que los alumnos trabajan juntos para maximizar su propio aprendizaje y el de los demás. (p. 3).

Asimismo, la disposición de los tiempos son determinantes en los grupos de aprendizaje para lograr objetivos comunes ante un trabajo cooperativo. Johnson, Johnson y Holubec (1999) sostiene que "El aprendizaje cooperativo comprende tres tipos de grupos de aprendizaje: formales, informales y de base" (p. 3).

Según, Chomsky (2000): a los estudiantes "no hay que verlos como un simple auditorio, sino como elemento integrante de una comunidad con preocupaciones compartidas, en la que uno espera poder participar constructivamente" (p. 29).

Teniendo en cuenta lo mencionado párrafos arriba, se puede inferir; aquel que emplea reiteradamente los grupos formales, los informales y los de base adquirirá un grado tal de práctica que podrá estructurar situaciones de aprendizaje cooperativo en forma automática, sin tener que idearlas ni planificarlas conscientemente. Podrá entonces utilizar correctamente el aprendizaje cooperativo durante todo el resto de su actividad docente. Del mismo modo nos permite identificar los elementos básicos del aprendizaje cooperativo, que viene hacer la interdependencia positiva, interacción cara a cara, responsabilidad individual, técnicas interpersonales y de equipo (habilidades y estrategias) y evaluación grupal.

Del mismo modo, el aprendizaje cooperativo ofrece mayores posibilidades para aprender debido a las oportunidades que proporciona de explicar y razonar los diferentes puntos de vista de los alumnos/as y por la capacidad que tienen los alumnos para entender las dificultades de los compañeros.

Por tanto, en los contextos cooperativos no sólo es importante lo que se aprende, sino también cómo se aprende. Incluso en muchas ocasiones se pone el énfasis en los procesos más que en los resultados, ya que se apunta a que el alumno aprenda a aprender desde un enfoque socio-constructivista. Piaget, Vygotsky y Ausubel, entienden que el desarrollo se produce por medio de la interacción social, así, el aprendizaje cooperativo es visto como una alternativa a la educación tradicional.

Por otra parte, con respecto a la formación docente por competencia, Tobón, Pimienta y García (2010), mencionan que:

La formación de las competencias como parte de la formación humana integral, a partir del proyecto ético de vida de cada persona, dentro de escenarios educativos colaborativos y articulados con lo social, lo económico, lo político, lo cultural, el arte, la ciencia y la tecnología. (p. 8). 
En tal sentido, “Una competencia, entonces, no es sólo tener un saber hacer, un saber conocer o un saber ser por separado, sino movilizar los diversos saberes (ser, hacer y conocer) hacia el logro de una meta determinada en el contexto". (Tobón, Pimienta y García, 2010, p. 12)

Con respecto al rol docente:

El contexto social actual y los cambios que se avecinan en el futuro cercano nos plantean el reto de pasar del énfasis en la planificación de la enseñanza, a un nuevo papel docente, que conlleva la generación de situaciones significativas, con el fin de que los estudiantes aprendan lo que requieren para su autorrealización y su participación en la sociedad. (Tobón, Pimienta y García, 2010, p. 20).

Las competencias implican necesariamente la relación de la teoría con la práctica, la movilización de conocimientos, el rescate de conocimientos previos de experiencias escolarizadas o no escolarizadas que se relacionan o son aplicables a una situación, también es determinante la individualidad y personalidad del estudiante, la actitud con la que afrontará la situación, los valores que rigen su actuar y cómo se va a proyectar ante alguna circunstancia; la actitud crítica para identificar la solución o posibles alternativas, si las hay y para afrontar el caso y el proceso de toma de decisiones; así como la ejecución de las conductas reflejo de todo lo anterior que conlleva a la situación presentada. (Castellanos, Morga y Castellanos, 2013, p. 22).

Teniendo en cuenta el aspecto educativo:

La educación basada en competencias se habla de un aprendizaje constante y permanente, que, contextualizado, permite la formación con conocimientos actualizados y útiles para el desarrollo integral de las personas a lo largo de su vida, de manera intencionada y planificada. (Castellanos, Morga y Castellanos, 2013, p. 24).

Considerando las variables de estudio analizadas, los referentes teóricos, explican que las actividades en equipos cooperativos permiten mejorar las competencias desde un punto de vista global, las competencias genéricas y específicas.

Por otra parte, cabe mencionar que, en el proceso de investigación se tuvo en consideración las variables y su respectiva operacionalización: Variable 1: Aprendizaje cooperativo, teniendo como dimensiones e indicadores (interdependencia positiva: miembro de su grupo, vínculo existente; interacción cara a cara: interacción con responsabilidad, apoyo mutuo, intercambio de aprendizaje; responsabilidad individual: logro de metas, participación individual, habilidades interpersonales: habilidades para organizarse en equipo, confianza en el grupo, y evaluación grupal: logro de aprendizaje), Variable 2: Formación docente por competencias, teniendo como dimensiones e indicadores (cognitivas: pensamiento crítico, creatividad e innovación, resolución de problemas, comunicación asertiva, manejo de la información, apropiación de las tecnologías digitales; Intrapersonales: pensamiento reflexivo, habilidades personales; e interpersonales: colaboración, responsabilidad social). 


\section{Metodología}

Permitió los procedimientos totalmente precisa, teniendo en cuenta los principios básicos del método científico. Por su naturaleza, la investigación fue de tipo básico, porque estuvo orientada a la búsqueda de nuevos conocimientos, recogiendo información de la realidad, en un esfuerzo por conocer mejor el problema investigado; y de nivel: descriptivo y correlacional. Se detalló las características de las variables y de por qué se presentan de tal o cual modo, es correlacional, porque se establece la relación entre las variables investigadas.

La investigación fue realizada con diseño correlacional. Al respecto, Hernández, Fernández y Baptista (2014) refieren que el diseño correlacional: "Describen relaciones entre dos o más categorías, conceptos o variables en un momento determinado" (p. 157). Que permitió utilizar el nivel y tipo de investigación sobre la muestra determinada y la relación entre las variables. Los Instrumentos de recolección de información, fueron conformados por: fichas de recojo de información y el cuestionario de 20 preguntas, que se empleó en los estudiantes de forma individual de acuerdo a la muestra determinada, donde se consideraron a las variables y sus respectivos indicadores, lo cual fueron aplicados a los estudiantes, de acuerdo a la muestra determinada.

La población estuvo conformada por 1169 estudiantes de la Facultad de Ciencias de la Educación de la Universidad Nacional Daniel Alcides Carrión, de la que se extrajo una muestra representada por 86 estudiantes, utilizando de tipo de muestra no aleatoria a criterio del investigador.

El método principal o método científico, garantizó el manejo de la adecuada secuencia lógica entre problema, objetivo, indicadores; utilizando en todo momento el sustento teórico. El método específico utilizado, Ex - Post - Facto cuyo propósito es investigar relaciones de causa - efecto observando hechos o manifestaciones que tendrán lugar, de cosas o acontecimientos ya ocurridos. A partir de una situación actual, se indaga hacia atrás, para identificar datos disponibles, hechos y posibles factores causales. Se trata de analizar la evolución y tendencia de ciertos hechos en el pasado y en el presente, lo que ayudaría para predecir su comportamiento en un futuro (Sánchez, 1998). En este proceso se utilizó las técnicas: observación directa y el cuestionario (se aplicó el instrumento validado a cada estudiante); revisión bibliográfica (visita a la biblioteca de la universidad y de la revisión infografía).

\section{Resultados y discusión}

Para obtener la relación entre los variables se usó el paquete estadístico SPSS versión 19, se aplicó el estadígrafo estadístico de Rho de Spearman. Teniendo en cuenta los criterios siguientes:

a. Fórmula para determinar la correlación:

$$
\text { Correlación de pearman } \quad r_{s}=1-\frac{6 \sum_{i=1}^{n} d_{i}^{2}}{n^{3}-n} \quad-1 \leq r s \leq+1
$$

b. Los intervalos:

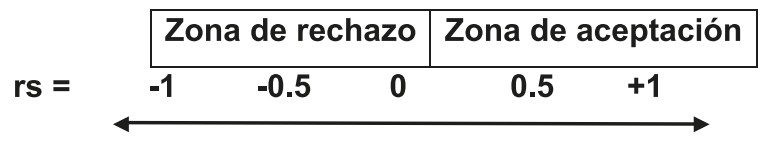


c. Rango Relación: 0,4 - o,69: correlación positiva moderada.

d. Resultado estadístico:

Correlaciones

\begin{tabular}{|c|c|c|c|c|}
\hline & & & Aprendizaje & Formación \\
\hline \multirow{2}{*}{ Rho de Spearman } & Aprendizaje & $\begin{array}{l}\text { Coeficiente de correlación } \\
\text { Sig. (bilateral) } \\
\mathrm{N}\end{array}$ & $\begin{array}{l}1,000 \\
86 \\
\end{array}$ & $\begin{array}{l}, 64^{8^{*} \star} \\
, 000 \\
86 \\
\end{array}$ \\
\hline & Formación & $\begin{array}{l}\text { Coeficiente de correlación } \\
\text { Sig. (bilateral) } \\
\mathrm{N}\end{array}$ & $\begin{array}{l}, 648^{* *} \\
, 000 \\
86\end{array}$ & $\begin{array}{l}1,000 \\
86\end{array}$ \\
\hline
\end{tabular}

**. La correlación es significativa al nivel o,01 (bilateral).

Como el valor de rs $=0.648$ y esto es $>$ o, la correlación es significativa, positiva moderada, por lo tanto, existe relación significativa entre aprendizaje cooperativo y la formación docente por competencia en la Universidad Nacional Daniel Alcides Carrión.

Teniendo en cuenta el resultado obtenido en la investigación, en el cual se demuestra que, existe relación significativa entre aprendizaje cooperativo y la formación docente por competencia en la Universidad Nacional Daniel Alcides Carrión; además, algunas conclusiones de la literatura revisada para esta investigación: Martínez (2016) menciona que, cuando los estudiantes trabajan de manera cooperativa en una asignatura de idioma extranjero, no solo aprenden contenidos porque las actividades cooperativas requieren que los estudiantes participen activamente en el proceso de enseñanza-aprendizaje y que adquieran las competencias que necesitarán en el mercado laboral; así también, Carbajal (2017) manifiesta en una de sus conclusiones que, existe relación positiva entre aprendizaje cooperativo y competencias genéricas en el estudiante de la Universidad Nacional Mayor de San Marcos, Lima 2017.

Considerando, el resultado y las conclusiones descritas y no habiendo en ellos posiciones que contradicen al resultado obtenido en la investigación; se corrobora que, existe relación significativa entre aprendizaje cooperativo y la formación docente por competencias en la Universidad Nacional Daniel Alcides Carrión.

\section{Conclusiones}

La metodología del aprendizaje cooperativo se relaciona con la formación docente por competencias, lo cual nos permite obtener resultados significativos en cuanto a los aprendizajes en la Universidad Nacional Daniel Alcides Carrión.

Los elementos básicos del aprendizaje cooperativo, son importantes durante la formación docente por competencia en el desarrollo de habilidades profesionales.

El aprendizaje cooperativo nos permite desarrollar la formación docente por competencia en lo cognitivo, en lo intra e interpersonal.

En la investigación la correlación es significativa y positiva moderada, lo cual nos permite recalcar la importancia del aprendizaje cooperativo en la formación docente por competencia. 


\section{Referencias bibliográficas}

Carbajal, J. (2017). El aprendizaje cooperativo y las competencias genéricas en el estudiante de la Universidad Nacional Mayor de San Marcos, Lima 2017. (Tesis de maestría, Universidad Cesar Vallejo). En: http://repositorio.ucv.edu.pe/bitstream/handle/ UCV/17025/Carbajal_ LJ.pdf? sequence=1\&isAllowed=y

Castellanos, N., Morga, L. y Castellanos, A. (2013). Educación por competencias: hacia la excelencia en la formación superior. Mexico. Red Tercer Milenio.

Ceinos, C. y García, R. (2009). El aprendizaje cooperativo como propuesta de innovación en el proceso de enseñanza aprendizaje: descripción de una experiencia. Recuperado desde: http://www. educacion.udc.es/grupos/gipdae/documentos/congreso/xcongreso/pdfs/t3/t3c67.pdf

Chomsky, N. (2000). La (des)educación. En: https://auladefilosofia.net/2015/05/18/ noamchomsky-la-deseducacion-200o/

Hernández, R. Fernández, C., y Baptista, M. (2014). Metodología de la Investigación. México: McGraw-Hill

Johnson, D., Johnson, R. y Holubec, E. (1999). El aprendizaje cooperativo en el aula. Buenos Aires. Editorial Paidós. En: http://conexiones.dgire.unam.mx/wp-content/uploads/2017/og/Elaprendizaje-cooperativo-en-el-aula-Johnsons-and-Johnson.pdf

Martínez, M. (2016). Cómo utilizar el aprendizaje cooperativo para evaluar las competencias emocionales de los estudiantes: un ejemplo práctico en el nivel terciario. Problemas de perfil en el desarrollo profesional de los docentes. En: http://www.scielo.org.co/pdf/prf/v18n2/ v18n2a10.pdf

Rué, J. (2012). Bases del trabajo cooperativo [Archivo PDF]. En: https://ikaskide.files.wordpress. com/2012/04/j-ruc3a9-ice-giac-bases-del-trabajo-cooperativo.pdf

Sánchez, H. (1998). Metodología y diseños en la investigación científica. Lima, Perú: Mantaro.

Tobón, S., Pimienta, J. y García, J. (2010). Secuencias didácticas: aprendizaje y evaluación de competencias. México. Pearson educación.

(C) Los autores. Este artículo es publicado por la Horizonte de la Ciencia de la Unidad de Posgrado de la Facultad de Educación de la Universidad Nacional del Centro del Perú. Este es un artículo de acceso abierto, distribuido bajo los términos de la Licencia Atribución-No Comercial 4.0 Internacional.(https://creativecommons.org/licenses/by-nc/4.o/), que permite el uso no comercial y distribución en cualquier medio, siempre que la obra original sea debidamente citada. 